\title{
Acute stress-induced cortisol elevation during memory consolidation enhances pattern separation
}

\author{
Alice Jiang, ${ }^{1}$ Tammy T. Tran, ${ }^{1}$ Farrah N. Madison, ${ }^{2}$ and Arnold Bakker ${ }^{3}$ \\ ${ }^{1}$ Department of Psychological and Brain Sciences, Johns Hopkins University School of Arts and Sciences, Baltimore, Maryland 21218, \\ USA; ${ }^{2}$ Department of Psychology, University of Maryland, College Park, Maryland, 20742, USA; ${ }^{3}$ Department of Psychiatry and \\ Behavioral Sciences, Johns Hopkins University School of Medicine, Baltimore, Maryland 21287, USA
}

\begin{abstract}
Stress is a potent modulator of brain function and particularly mnemonic processes. While chronic stress is associated with long-term deficits in memory, the effects of acute stress on mnemonic functions are less clear as previous reports have been inconsistent. Some studies suggest that cortisol, a stress hormone that modulates biological changes in response to stress, may enhance memory consolidation and impair memory retrieval. However, other studies report no effect of cortisol on either memory consolidation or retrieval. These discrepancies could be due to differences in the timing and sequencing of the experimental procedures or individual differences in participants' stress response. In the present study, we examined the effect of increased cortisol levels due to acute stress, induced by the Trier Social Stress Test (TSST), on a pattern separation memory task while differentiating the distinct stages of memory processing and controlling for the effects of diurnal variation. Sixty-nine young adults completed a 2-d study in which subjects either underwent the TSST immediately following the encoding part of the memory task, targeting memory consolidation, or immediately prior to the recognition part of the memory task on the second day, targeting memory retrieval. Control subjects completed the same study procedures but underwent a control version of the TSST that did not induce a stress response. Mnemonic discrimination of highly similar stimuli was enhanced by stress induced during consolidation with better discrimination showing a significant correlation with increased cortisol responses. Stress induced during memory retrieval showed no significant effect on memory performance. These findings suggest that stress induced changes in cortisol differentially affect the consolidation and retrieval stages of memory function.
\end{abstract}

Extensive evidence has suggested that stress is a potent modulator of brain function and cognition, affecting attention, sensory, and particularly mnemonic processes (de Kloet et al. 1999; McEwen 1999; Sandi 2003; Sandi and Pinelo-Nava 2007). While chronic stress, or prolonged activation of the stress response, is associated with long-term deficits in memory (for review, see Joëls et al. 2004), acute stress has been observed to have differential effects on mnemonic functions. Glucocorticoids (cortisol in humans, corticosterone in rats) are stress hormones that modulate changes in the body that occur in response to stress. In healthy adults, several studies have suggested an enhancing effect of cortisol on memory consolidation, the process of converting memories into long-term storage (Smeets et al. 2008; McCullough and Yonelinas 2013; for review, see de Quervain et al. 2009) and a detrimental effect of stress on memory retrieval (Roozendaal, 2002; de Quervain et al. 2003; Coluccia et al., 2008). However, others have failed to find such beneficial and detrimental effects of stress on consolidation and retrieval (Rimmele et al. 2003, 2015; Boehringer et al. 2010).

One possible explanation for these conflicting results is that the effect of stress is nonlinear and instead follows an inverted U-shape dose response curve where moderate stress levels are associated with improved memory performance compared to very low or high levels of stress (Kovacs et al. 1976; Flood et al. 1978; Andreano and Cahill 2006). An alternative explanation is provided by the significant differences observed by Het et al. (2005) between studies that administered cortisol in the morning compared to the afternoon, likely due to diurnal changes in corti-

\section{Corresponding author: abakker@jhu.edu}

Article is online at http://www.learnmem.org/cgi/doi/10.1101//m.048546.118. sol concentrations and the timing of glucocorticoid treatment (e.g., before learning, after learning, or before retrieval) suggesting acute stress may affect learning, consolidation, and retrieval differently. Upon the induction of stress, peaks in cortisol levels typically occur $10 \mathrm{~min}$ after cessation of the stress exposure (Smeets et al. 2008; Het et al. 2009; Petrowski et al. 2010), although peaks have also been observed 20-25 min after the beginning of stress induction (Rohleder et al. 2001). The delayed peak response could cause elevated glucocorticoids to act on multiple memory processes if recall was tested immediately following new learning.

The present study aimed to investigate the effects of acute stress on hippocampus-dependent memory function by differentiating between the distinct stages of memory processing while controlling for the effects of diurnal variation. The Trier Social Stress Test (TSST), a standardized laboratory protocol that combines public speaking, mental arithmetic, threat anticipation, and social evaluation, was used to induce a robust and reliable acute stress response in participants either immediately following encoding or immediately preceding retrieval 24-h later (Kirschbaum et al. 1993). Memory performance was assessed using the object version of the behavioral pattern separation (BPS-O) task designed to tax hippocampus-dependent memory function (Kirwan and Stark 2007; Bakker et al. 2008; Stark et al. 2013). The BPS-O consists of an encoding phase and a retrieval phase facilitating the examination of differential effects of stress on memory consolidation and

(C) 2019 jiang et al. This article is distributed exclusively by Cold Spring Harbor Laboratory Press for the first 12 months after the full-issue publication date (see http://learnmem.cshlp.org/site/misc/terms.xhtml). After 12 months, it is available under a Creative Commons License (Attribution-NonCommercial 4.0 International), as described at http://creativecommons.org/licenses/by-nc/4.0/. 
retrieval. The task assesses pattern separation, the ability to differentiate highly similar experiences into distinct nonoverlapping representations, a process thought to rely on the dentate gyrus (DG) subregion of the hippocampus and crucial to reducing interference in the formation of new episodic memories. Balanced against pattern separation is pattern competition, a process enabling the retrieval of previously stored information based on commonalities between the current input and prior experiences. Pattern completion is thought to rely on the significant auto-associative collaterals of the CA3 subregion of the hippocampus. The significance of pattern separation and pattern completion in episodic memory function has been previously examined in rodents (Leutgeb et al. 2004, 2007; Wilson et al. 2005), normal memory function in humans (Bakker et al. 2008), age-related memory impairment in older adults (Yassa et al. 2010; Huffman and Stark 2017; Stark and Stark 2017) and patients with amnestic mild cognitive impairment (Bakker et al. 2012, 2015; Tran et al. 2017). While only an inferential measure, this behavioral task is thought to provide a measure of DG and CA3-mediated memory function which has been shown to be sensitive to stress and aging (Joels et al. 2008; Stark et al. 2013; for review, see Fa et al., 2014).

These methods were used to test the hypothesis that acute stress enhances the consolidation but impairs the retrieval of highly similar stimuli. Results show that acute stress induced during memory consolidation led to improved discrimination accuracy and better recollection of detailed memory across varying degrees of similarity. Measures of cortisol showed a positive linear relationship suggesting that increased cortisol levels are associated with stronger consolidation of separated representations of highly similar items. In contrast, stress induced during the retrieval phase did not significantly impact memory performance for similar items when compared to controls. These findings show that increased cortisol levels resulting from an acute stress response affect specifically the consolidation phase of memory encoding, enhancing the encoding of pattern separated information thereby increasing the level of detail available for retrieval while the retrieval process itself is not affected by acute changes in cortisol levels.

\section{Materials and Methods}

\section{Participants}

Eighty healthy young adult males participated in the study. Female participants were excluded to avoid potential confounds due to menstrual cycle phases and use of hormonal contraceptives (Kirschbaum et al. 1999; Bouma et al. 2009). Inclusion criteria required all participants to be between the ages of 18 and $30 \mathrm{yr}$ old, native English speakers, and able to provide written informed consent. Exclusion criteria prevented individuals who met any of the following characteristics from participating in the study: major psychiatric or behavioral disorders including current major depression, major neurological conditions, or the presence of clinically significant disease. These criteria were evaluated through interviews and self-report.

The study was approved by the Institutional Review Board of the Johns
Hopkins Medical Institutions. All participants provided written informed consent and were compensated with class credit for their participation in the study.

\section{Study design and procedures}

Participants were asked to complete two visits over the course of $2 \mathrm{~d}$ and were randomly assigned to one of four groups: consolidationstress, consolidation-control, retrieval-stress, or retrieval-control (Fig. 1). In the consolidation-stress group, exposure to stress was aimed to specifically influence the conversion of short-term memories into long-term memories (memory consolidation). Participants in the consolidation-stress group first completed the encoding phase of behavioral pattern separation objects memory task (BPS-O; see 2.3), which presented images of objects for the participant to study. Immediately afterwards, participants were exposed to a stressful situation through the TSST. In the second visit which occurred $24 \mathrm{~h}$ after the first visit, participants completed the recognition phase of the BPS-O task, which tested the participant's memory for the images seen the day before.

In the retrieval-stress group, exposure to stress was aimed to influence the process of retrieving long-term memories (memory retrieval). Participants in the retrieval-stress group completed the encoding phase of the BPS-O task during their first visit. After a 24-h delay they were exposed to a stressful situation with the TSST and completed the recognition phase of the BPS-O memory task immediately afterwards.

All control participants in the study completed the same procedures except with a nonstressful, control version of the TSST (control-TSST). Of the control participants, half were exposed to the control-TSST after the encoding phase of the memory task (consolidation-control group) while the other half were exposed
A Consolidation$(n=21)$

Retrieval-
stress group
$(n=20)$

B

Day 1: Encoding Task (Outdoor/Indoor)
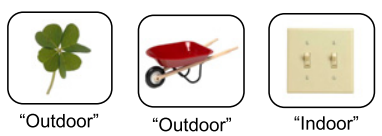

Day 2: Recognition Task (Old/Similar/New)
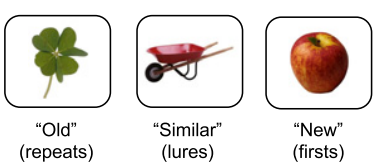

C

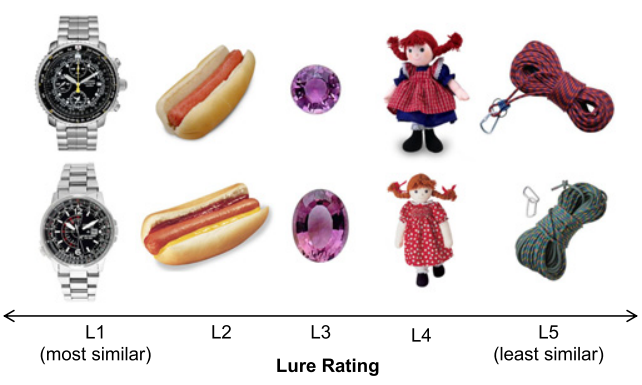

Figure 1. (A) Outline of study design. Participants in the consolidation-stress group completed the encoding phase of the memory task followed by the TSST during the first visit. During the second visit, $24 \mathrm{~h}$ later, participants completed the recognition phase of the memory task. Participants in the retrieval-stress group completed the encoding phase of the memory task during their first visit, and then were exposed to the TSST and recognition phase of the memory task in the second session. Consolidation-control and retrieval-control groups followed the same sequence and timing of procedures as the corresponding experimental groups but completed a nonstressful control-TSST instead. (B) Behavioral pattern separation task. On the first day, participants completed an incidental indoor-outdoor judgment task during the encoding phase. Twenty-four hours later, recognition was tested using an old-similar-new judgment task with repeat, first, and lure images. (C) Sample stimuli and their lures arranged in order of mnemonic similarity from most similar (L1) to least similar (L5). 
prior to the recognition phase of the BPS-O memory task (retrievalcontrol group).

All participants were asked to provide saliva samples immediately before and after the TSST or control-TSST. Finally, participants completed a self-report questionnaire (the State Trait Anxiety Inventory) to assess the subjective level of psychological stress (Spielberger and Sydeman 1994). Following completion of all the study procedures, participants were debriefed about the purpose of the study.

\section{Behavioral pattern separation memory task}

The behavioral pattern separation task-object version (BPS-O) is a computer-based memory task designed to tax hippocampusdependent memory function. The task has been studied extensively and used in both normal and clinical populations (Kirwan et al. 2007; Bakker et al. 2008, 2012, 2015; Stark et al. 2013, 2015; Tran et al. 2017). The BPS-O task consists of an incidental encoding phase and a three-alternative forced-choice recognition phase (Fig. 1B). In the incidental encoding phase, participants passively encoded pictures of everyday objects by determining whether the observed item is most commonly an indoor or an outdoor item (128 items shown for $2.5 \mathrm{sec}$ each, with an inter-stimulus interval (ISI) of $0.5 \mathrm{sec}$ ). In the recognition phase performed $24 \mathrm{~h}$ later, participants were tested on whether images they saw were "old," "similar," or "new" to the images they saw in the study phase (192 items total, $2.5 \mathrm{sec}$ each, $0.5 \mathrm{sec}$ ISI). One-third of the images (64 images) were exactly the same from the study phase (repeats), onethird of the images were similar but not identical to those seen during the study phase (lures), and one-third of the images were novel images seen for the first time (firsts). The critical trials were the lure trials assessed by the rates at which the participant correctly identified lure items as "similar." Correct identification of the merely similar lure items is thought to depend on DG mediated pattern separation, referring to the ability to separate overlapping inputs into distinct representations. Incorrectly identifying lures as "old" is thought to reflect evidence of pattern completion, a complementary function thought to be mediated by the CA3 and its strong auto-associative network, reflecting retrieval of previously stored information based on commonalities between the current input and prior experiences. Based on the probability of calling lures "old" in a previous study, lure items were further divided into five bins ranging from most mnemonically similar lures (L1) to least mnemonically similar lure (L5) (Fig 1C; Lacy et al. 2011).

\section{Trier Social Stress Test}

The TSST is designed to provide a standardized exposure to stress in a laboratory setting (Kirschbaum et al. 1993). The component of the TSST designed to induce stress is divided into three 5-min parts. In the first part, participants were asked to prepare a speech explaining why they were the best candidates for an ideal job. Participants were allowed to use pen and paper during a preparation period, but were not allowed to use their notes during the presentation. In the second part, participants presented their speech to a panel of two researchers, one male and one female, posing as expert judges. The judges maintained neutral expressions and observed the participant without comment. If the participants did not use the full $5 \mathrm{~min}$ allocated for this portion of the test, the judges asked the participants to continue until time was up. In the final part of the TSST, participants performed a mental arithmetic task by serially subtracting the number 13 from 3087. If a mistake was made, the judges asked the participants to start again from the beginning. The TSST has been shown to reliably induce stress in participants and meta-analysis suggests that the TSST is the most useful and appropriate standardized protocol for studies of stress hormone reactivity (Dickerson and Kemeney 2004).

Prior to and immediately following the stressful portion of the TSST, participants were given a 10-min rest period and 10-min recovery period. The rest period at the beginning of the TSST gave participants a chance to reach baseline hormone levels for an accurate pre-TSST cortisol measurement. Cortisol has a delayed release, so the recovery period and post-TSST tasks were scheduled to coincide with the peak hormonal response.

The control version of the TSST was designed to be as similar as possible to the TSST, but without the stressful components, for example specifically lacking the social evaluative threat (Het et al. 2009). The control-TSST was performed in the same room as the TSST. Instead of preparing a speech to give in front of a panel of judges, the control-TSST gave participants $5 \mathrm{~min}$ to prepare for a 5-min talk about a movie, novel, or recent holiday trip. Participants were told that they would speak alone in an empty room, and were given paper and pen to prepare and use throughout the task. The researcher only entered the room between each part to give instructions to the participants. After preparing and talking on the topic of their choice, participants were asked to begin serially adding by increments of 15 , starting at 0 . The researcher, once again, left the participants alone to complete the task, and controlled for the participants' compliance by asking them for the final number reached in the serial addition.

\section{Saliva sampling and biochemical analysis}

In order to minimize differences in baseline cortisol levels and enable adequate saliva sampling for cortisol assessment, participants were asked to refrain from consuming alcohol or any recreational drugs $12 \mathrm{~h}$ before each session; heavy physical activity and caffeinated drinks $3 \mathrm{~h}$ before each session; and food, noncaffeinated drinks, tooth brushing/flossing, and smoking $1 \mathrm{~h}$ before each session. To reduce the impact of diurnal variation in cortisol levels, all testing was performed in the afternoon between 3:00 and 6:00 p.m., when hormone levels are relatively stable. Upon arrival, participants were asked to rinse their mouth with water to prevent potential contamination of the saliva sample.

Saliva samples were collected to obtain free cortisol levels, a marker of hypothalamic-pituitary-adrenal axis activity. Saliva samples were collected by the passive drool method into $2 \mathrm{~mL}$ cryovials. Samples were kept at $-20^{\circ} \mathrm{C}$ until they were analyzed. Saliva was assayed for salivary cortisol concentrations using a commercially available immunoassay kit (Salimetrics). The minimal concentration of cortisol that could be distinguished from 0 was $0.007 \mu \mathrm{g} / \mathrm{dL}$. The intra-assay coefficient of variation $(n=38)$ was $3.7 \%$, and the inter-assay coefficient of variation $(n=5)$ was $10.2 \%$.

\section{Results}

\section{Statistical analysis}

To ensure that participants understood and completed the memory task as instructed, the accuracy of identifying novel (first) and repeated images was examined. Eleven participants (two from the consolidation-stress group, two from consolidation-control group, four from retrieval-stress group, and three from retrieval-control group) were excluded from data analysis due to poor memory task performance, which was defined as scoring below a 33\% (chance) correct response rate for foil or repeat images.

Seven participants (three from the consolidation-stress group, two from consolidation-control, one from retrieval-stress, and one from retrieval-control) were further excluded from cortisol data analysis due to undetectable salivary cortisol levels in the samples collected either pre- or post-TSST administration. After removing these participants, the consolidation-stress group included 21 participants, the consolidation-control group included 10 participants, retrieval-stress group included 20 participants, and retrievalcontrol group included 11 participants.

First, differences between the two control subgroups (consolidation-control and retrieval-control) were examined for differences in age $\left(t_{(22)}=0.779, P=0.44\right)$, pre-TSST STAI score $\left(t_{(22)}=\right.$ $1.544, P=0.14)$, post-TSST STAI score $\left(t_{(22)}=0.983, P=0.34\right)$, pre-TSST cortisol levels $\left(t_{(22)}=0.130, P=0.90\right)$, and post-TSST cortisol levels $\left(t_{(22)}=0.020, P=0.98\right)$, using independent samples $t$-tests. BPS-O task performance was also compared between the two 
Table 1. Demographics, clinical characterization, and task performance of study participants

\begin{tabular}{|c|c|c|c|c|c|c|c|c|}
\hline \multirow[b]{2}{*}{ Characteristic } & \multicolumn{3}{|c|}{ Consolidation-stress $(n=24)$} & \multicolumn{3}{|c|}{ Retrieval-stress $(n=21)$} & \multicolumn{2}{|c|}{ Controls $(n=24)$} \\
\hline & Mean & SD & $P$-value & Mean & SD & $P$-value & Mean & SD \\
\hline Age & 19.13 & 1.26 & 0.46 & 19.95 & 1.32 & 0.40 & 19.58 & 1.56 \\
\hline Pre-STAI score & 35.58 & 9.33 & 0.65 & 35.48 & 7.83 & 0.65 & 34.46 & 7.48 \\
\hline Post-STAI score & 51.88 & 12.14 & $<0.001$ & 50.62 & 9.78 & $<0.0001$ & 35.79 & 6.43 \\
\hline Pre-CORT $(\mu \mathrm{g} / \mathrm{dL})$ & 0.13 & 0.11 & 0.36 & 0.20 & 0.16 & 0.55 & 0.17 & 0.18 \\
\hline Post-CORT ( $\mu \mathrm{g} / \mathrm{dL})$ & 0.28 & 0.16 & 0.047 & 0.44 & 0.25 & 0.0002 & 0.18 & 0.18 \\
\hline Repeats called "old" & 0.60 & 0.14 & 0.39 & 0.64 & 0.14 & 0.92 & 0.63 & 0.13 \\
\hline Lures called "similar" & 0.47 & 0.09 & $<0.01$ & 0.30 & 0.07 & 0.29 & 0.33 & 0.11 \\
\hline Firsts called "new" & 0.68 & 0.18 & 0.058 & 0.68 & 0.20 & 0.10 & 0.77 & 0.12 \\
\hline
\end{tabular}

control groups for correct identification of repeats $\left(t_{(22)}=0.318, P=\right.$ $0.75)$, lures $\left(t_{(22)}=0.130, P=0.90\right)$, and firsts $\left(t_{(22)}=0.166, P=0.87\right)$. As no significant differences between the control groups were observed, data from the two control groups were collapsed to form a single control group ( $n=24$ for behavioral data, $n=21$ for cortisol analysis) for the remainder of the analyses (Table 1). Differences in performance and cortisol levels between groups were examined using analysis of variance (ANOVA). Differences within group before and after administration of the TSST were examined using within sample $t$-tests.

\section{Effectiveness of stress induction}

Prior to administration of the TSST, self-report State Trait Anxiety Inventory (STAI) scores did not show a difference between the control group, consolidation-stress group, and retrieval-stress group $\left(F_{(2,66)}=0.72, P=0.49\right)$ in a one way-ANOVA. Analysis of the STAI scores before and after administration of the TSST revealed that the TSST successfully induced subjective psychological stress (Fig. 2A). A two-way repeated measures ANOVA of group (consolidation-stress and control) and response (pre- and post-STAI scores), showed a significant interaction between group and pre- and poststress STAI scores $\left(F_{(1,46)}=65.5, P<0.001\right)$. A separate two-way repeated measures ANOVA of group and response similarly also showed an interaction between the retrieval-stress group and the control group in pre- and post-stress STAI scores $\left(F_{(1,43)}=\right.$ 29.92, $P<0.0001)$. Planned post-hoc analysis showed that 10 min after administration of the TSST, STAI scores were significantly higher in the consolidation-stress group $\left(t_{(23)}=5.73, P<\right.$ $0.0001)$ and retrieval-stress group $\left(t_{(20)}=6.37, P<0.0001\right)$ compared to STAI scores within the same group before the administration of the TSST. Participants in the control conditions did not exhibit an increase in subjective stress following the control-TSST $\left(t_{(23)}=1.18, P=0.25\right)$ using a repeated measures $t$-test.

To verify that the TSST also induced a physiological stress response, we compared the salivary cortisol levels between stress and control groups (Fig. 2B). Prior to the TSST, concentrations of salivary cortisol did not show a difference between the control group, consolidation-stress group, or retrieval-stress group $\left(F_{(2,59)}=1.218\right.$, $P=0.30)$. A two-way repeated measures ANOVA of group (consolidation-stress and control group) and cortisol levels, showed a significant interaction $\left(F_{(1,40)}=\right.$ $16.33, P<0.001)$ between group and preand post-stress cortisol levels. A separate two-way repeated measures ANOVA showed a similar interaction between the retrieval-stress group and the control group pre- and post-stress cortisol level $\left(F_{(1,39)}=23.38, P<0.0001\right)$. Planned posthoc analysis showed that 10 min after ad- ministration of the TSST, concentrations of salivary cortisol were significantly higher in the consolidation-stress group $\left(t_{(20)}=4.90\right.$, $P<0.001)$ and retrieval-stress group $\left(t_{(19)}=5.26, P<0.001\right)$ compared to the salivary concentrations within the same group before the administration of the TSST. Participants in the control conditions did not exhibit an increase in salivary cortisol following the control-TSST $\left(t_{(20)}=0.47, P=0.65\right)$ (Table 1$)$.

\section{Memory performance}

Participants identified repeats as "old" and firsts as "new" at similar rates across groups. This performance was consistent with a previous study using a similar population (Borota et al. 2014). To determine whether the ability to differentiate the critical lure items was affected by stress, we examined the proportion of lure stimuli correctly identified as "similar." Participants in the consolidationstress condition were significantly more likely to correctly call lure items "similar" compared to participants in the control condition, who were more likely to call lure items "old" $t_{(46)}=4.88, P<$ 0.0001 ) (Fig. 3A). The retrieval-stress group revealed there was no significant difference in lure responses between retrieval-stress and control groups $\left(t_{(43)}=1.08, P=0.29\right)$ (Fig. 3B).

We next considered whether pattern separation performance was affected by varying degree of similarity. The consolidationstress group correctly identified more lure items as "similar" compared to the control group across all lure bins, without any bias for high similarity or low similarity $\left(t_{(46)}=3.98, P=<0.001 ; t_{(46)}=\right.$ $2.36, P=0.02 ; t_{(46)}=2.21, P=0.03 ; t_{(46)}=6.35, P<0.001 ; t_{(46)}=$ $2.90, P<0.01$ for lure bins $1,2,3,4$, and 5, respectively; Fig. 4). The retrieval-stress group did not significantly differ from the control group in performance across all lure bins.

\section{Cortisol responses and memory}

We subsequently examined the relationship between stress-related changes in cortisol and memory performance by analyzing the
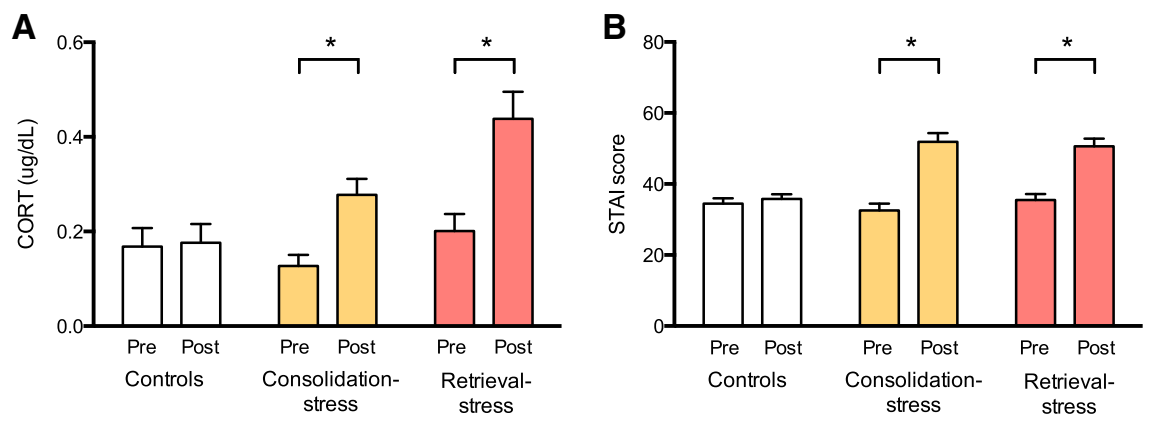

Figure 2. The TSST induces a psychological and physiological response to stress. Mean $(A)$ STAI scores and $(B)$ salivary cortisol concentrations are higher post-TSST for stress groups but not for control groups. Error bars represent standard error of the mean. $\left({ }^{*}\right) P<0.05$. 
A
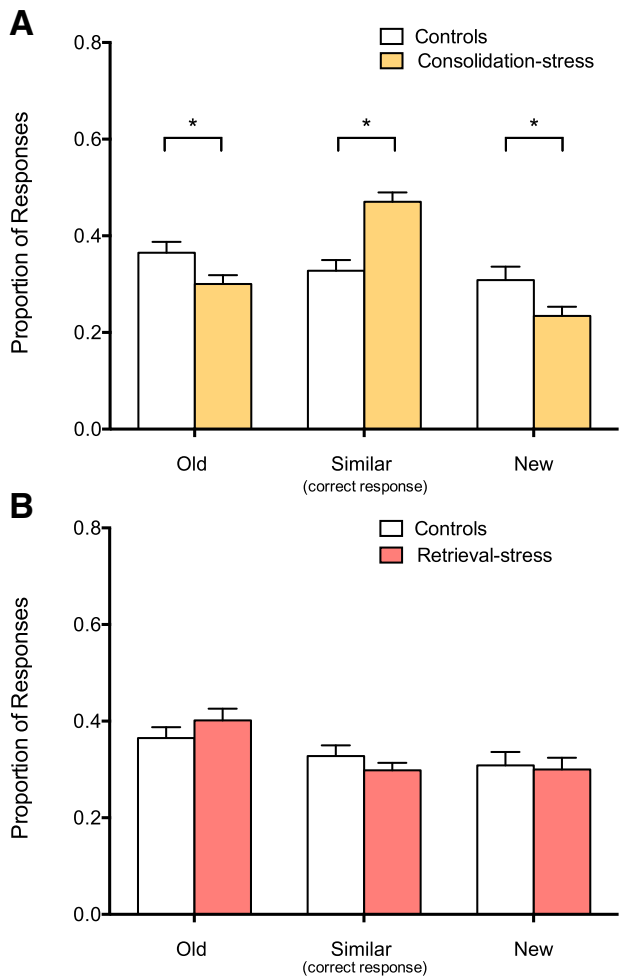

Figure 3. Increased stress during consolidation enhances memory retrieval $(A)$ The consolidation-stress group more often correctly called a lure item "similar" than "old." (B) The retrieval-stress group identified lure items at similar rates as controls. The mean probability of lures being called similar was $32.8 \%$ for the control group, $47.0 \%$ for consolidation-stress group and $29.8 \%$ for retrieval-stress group while the mean probability of foils called similar was $12.2 \%$ for the control group, $23.9 \%$ for consolidation-stress group, $14.5 \%$ for retrieval-stress group. Error bars represent standard error of the mean. $\left(^{*}\right) P<0.05$.

percent change in cortisol concentration between the pre- and post- samples against the proportion of correct responses to items on the behavioral pattern separation task for the consolidationstress and control group using a linear regression. No significant differences were observed for repeat items $\left(F_{(1,43)}=0.10, P=0.76\right)$ and first items $\left(F_{(1,43)}=0.01, P=0.92\right)$ between the percent change in cortisol concentration and the proportion of correct responses (Fig. 5A,C). However, for lure items, a significant positive, linear relationship was observed, such that a larger percentage increase in cortisol concentration was correlated with a higher proportion of correct responses in identifying lure items as "similar" $\left(F_{(1,43)}=\right.$ 4.642, $P=0.037$ ) (Fig. 5B).

\section{Discussion}

This study assessed the effects of acute stress during memory consolidation and retrieval in healthy young adults, specifically the role of cortisol in hippocampus-dependent memory function. The results show that acute stress during consolidation significantly enhances pattern separation performance and improves discrimination between highly similar stimuli. Participants in the consolidation-stress group more often correctly respond "similar" to lure items, indicative of enhanced pattern separation performance compared to control participants. This improved performance was observed for lure items of all degrees of mnemonic similarity equally. For the lure items, as accuracy increased, the proportion of incorrect responses in both the "new" and "old" catego- ries decreased, reflecting fewer misses and an improvement in memory accuracy overall. The findings reported here are consistent with previous reports of enhancing effects of cortisol on general memory performance (de Kloet et al. 1999; McGaugh and Roozendaal 2002) and extending those findings showing that cortisol specifically influences memory consolidation of highly similar stimuli across varying degrees similarity but does not influence memory retrieval in these young adults.

To assess the relationship between cortisol concentration and memory performance, we correlated the change in cortisol concentration and correct responses to each of the stimuli categories presented in the memory task (repeats, firsts, lures). Using a percent change in cortisol measure to account for natural variation and individual participant differences in baseline levels in cortisol concentrations and stress response (Smyth et al. 1998), we found no relationship between change in cortisol levels and performance for repeat and first items when cortisol is induced during consolidation. However, when cortisol is induced during memory consolidation, performance shows a positive linear correlation with change in cortisol with larger changes in cortisol associated with larger improvements in lure discrimination and pattern separation performance. The difficulty of this task combined with the delay and the stressful condition did result in lower performance, however this performance was consistent with other studies using a similar paradigm in a young adult sample (Borota et al. 2014).

Previous studies have suggested that cortisol has an effect only for emotionally arousing experiences and does not modulate neutral information (Kuhlmann and Wolf 2006; Holz et al. 2014). However, our results show an enhancing effect of cortisol on memory consolidation of neutral stimuli. As all stimuli included in the pattern separation task are designed to lack significant emotional valence, additional studies will be needed to consider the effect of emotional valence and arousal on pattern separation behavior. A recent meta-analysis by Shields et al. (2017) found evidence that post-encoding stress enhanced memory regardless of emotional valence. Identifying the role of valence in pattern separation memory tasks is particularly relevant in certain psychiatric conditions in which patients exhibit an impaired ability to distinguish between neutral and emotionally salient stimuli (Kheirbek et al. 2012).

In contrast to previous reports, these results also showed that participants in the retrieval-stress group did not perform significantly differently from the control group despite having elevated

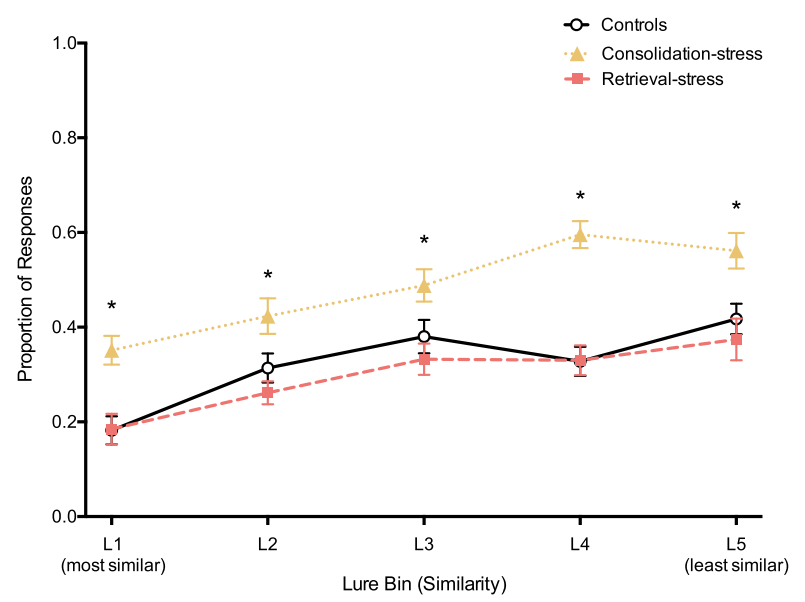

Figure 4. Increased stress during consolidation enhances memory across all similarity levels. Proportion of correct responses by lure bin (similarity) for each group. Error bars represent standard error of the mean. $\left(^{*}\right)$ $P<0.05$. 
A

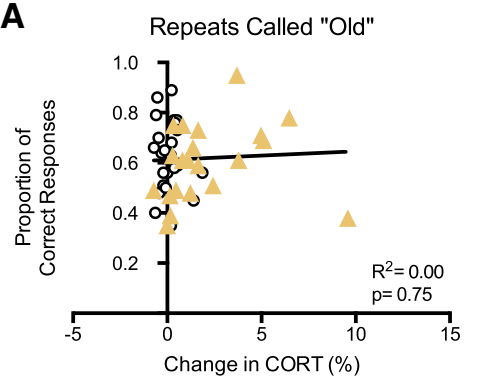

B

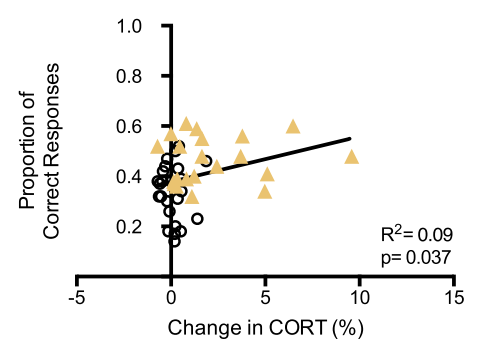

- Controls

Consolidation-stress
C

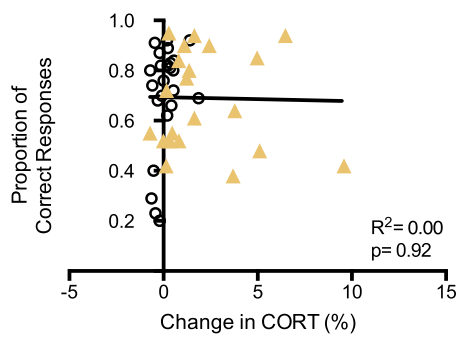

Figure 5. In the consolidation-stress group, change in cortisol concentration is correlated with memory performance for the critical lure items (B), but not for repeats called "old" $(A)$, or firsts called "new" $(C)$. Linear regression line shows slope across both groups.

cortisol levels immediately prior to the recognition memory task. In both animals and humans, several studies have reported impairing effects of glucocorticoids on memory retrieval (for reviews, see Wolf 2003; Het et al. 2005; de Quervain et al. 2009; Gagnon and Wagner 2016; Shields et al. 2017). However, elevated cortisol levels have also been observed to have little to no relationship with memory retrieval. Rimmele et al. (2015) reported that administration of a cortisol-suppressing drug led to significantly impaired free recall of emotional texts. Similarly, Boehringer et al. (2010) found that highly stressed participants with high arousal performed comparably to controls. These inconsistent results could be attributed to the differences between mineralocorticoid and glucocorticoid receptor activation in different hippocampal subregions (Harris et al. 2013; Rimmele et al. 2015). It has been previously shown in mice that chronic unpredictable stress causes adaptive plasticity to occur the most in the dorsal subregion (CA3 and DG) while hippocampal progenitor cells had decreased survival in the ventral subregion (CA1) (Hawley and Leasure 2012). Recently, Gagnon et al. (2018) reported a stress-induced decline in memory retrieval, associated with reduced posterior hippocampal activation on a functional neuroimaging task. Together, these results suggest that stress may impact specific hippocampal regions incongruously as well as affect specific memory processes that are dependent on those hippocampal subregions as observed in this study.

The present study recruited an all-male sample limiting the generalization of our findings. Females were excluded because they tend to exhibit smaller stress responses and more variable changes in salivary cortisol levels (Kajantie and Phillips 2006). Stress effects are also less consistently observed in females depending on menstrual cycle phases and the use of oral contraceptives (Kirschbaum et al. 1999; Bouma et al. 2009). Therefore, in this study, we chose to limit the population to only males, consistent with several previous reports (see Andreano and Cahill 2006; Henckens et al. 2009; McCullough et al. 2015). However, there is evidence suggesting that gender differences may moderate the link between stress and memory performance. Future studies should consider a sample that includes both genders in order to examine gender differences in cortisol modulation of memory performance.

Together, the results reported here provide evidence for the enhancing effect of cortisol on memory consolidation resulting in increased retrieval of detail memory. The modulating effects of cortisol observed in this study are likely mediated by the DG and CA3 subregions of the hippocampus known to be differentially affected by cortisol through different receptor densities. These findings suggest that induced stress will result in enhanced encoding of the context of the stressful experience likely reflecting an evolutionary advantage by providing a detailed representation of the objects or contexts to avoid without overgeneralizing this informa- tion. Mediation by specific hippocampal subregions also suggests that the interaction between cortisol and hippocampal-subregiondependent memory function may be altered in conditions where hippocampal subregions are affected by pathology and neurodegeneration. Future studies are needed to address these questions and further understand the role of the stress response on memory and cognition in healthy and pathological conditions.

\section{References}

Andreano JM, Cahill L. 2006. Glucocorticoid release and memory consolidation in men and women. Psychol Sci 17: 466-470. doi:10.1111/ j.1467-9280.2006.01729.x

Bakker A, Kirwan CB, Miller M, Stark CE. 2008. Pattern separation in the human hippocampal CA3 and dentate gyrus. Science 319: 1640-1642. doi:10.1126/science.1152882

Bakker A, Krauss GL, Albert MS, Speck CL, Jones LR, Stark CE, Yassa MA, Bassett SS, Shelton L, Gallagher M. 2012. Reduction of hippocampal hyperactivity improves cognition in amnestic mild cognitive impairment. Neuron 74: 467-474. doi:10.1016/j.neuron.2012.03.023

Bakker A, Albert MS, Krauss G, Speck CL, Gallagher M. 2015. Response of the medial temporal lobe network in amnestic mild cognitive impairment to therapeutic intervention assessed by fMRI and memory task performance. NeuroImage 7: 688-698. doi:10.1016/j.nicl.2015.02.009

Boehringer A, Schwabe L, Schachinger H. 2010. A combination of high stress-induced tense and energetic arousal compensates for impairing effects of stress on memory retrieval in men. Stress 13: 444-453. doi:10 $.3109 / 10253891003725256$

Borota D, Murray E, Keceli G, Chang A, Watabe JM, Ly M, Yassa MA. 2014 Post-study caffeine administration enhances memory consolidation in humans. Nat Neurosci 17: 201-203. doi:10.1038/nn.3623

Bouma EM, Riese H, Ormel J, Verhulst FC, Oldehinkel AJ. 2009. Adolescents' cortisol responses to awakening and social stress; effects of gender, menstrual phase and oral contraceptives. The TRAILS study. Psychoneuroendocrinology 34: 884-893. doi:10.1016/j.psyneuen.2009.01 .003

Coluccia D, Wolf OT, Kollias S, Roozendaal B, Forster A, Dominique JF. 2008. Glucocorticoid therapy-induced memory deficits: acute versus chronic effects. J Neurosci 28: 3474-3478. doi:10.1523/JNEUROSCI.4893-07 .2008

de Kloet ER, Oitzl MS, Joëls M. 1999. Stress and cognition: are corticosteroids good or bad guys? Trends Neurosci 22: 422-426. doi:10.1016/S0166-2236 (99)01438-1

de Quervain DJF, Henke K, Aerni A, Treyer V, McGaugh JL, Berthold T, Nitsch RM, Buck A, Roozendaal B, Hock C. 2003.

Glucocorticoid-induced impairment of declarative memory retrieval is associated with reduced blood flow in the medial temporal lobe. Eur J Neurosci 17: 1296-1302. doi:10.1046/j.1460-9568.2003.02542.x

de Quervain DJ, Aerni A, Schelling G, Roozendaal B. 2009. Glucocorticoids and the regulation of memory in health and disease. Front Neuroendocrinol 30: 358-370. doi:10.1016/j.yfrne.2009.03.002

Dickerson SS, Kemeny ME. 2004. Acute stressors and cortisol responses: a theoretical integration and synthesis of laboratory research. Psychol Bull 130: 355 . doi: $10.1037 / 0033-2909.130 .3 .355$

Fa M, Xia L, Anunu R, Kehat O, Kriebel M, Volkmer H, Richter-Levin G. 2014. Stress modulation of hippocampal activity-Spotlight on the 
dentate gyrus. Neurobiol Learn Mem 112: 53-60. doi:10.1016/j.nlm.2014 .04 .008

Flood JF, Vidal D, Bennett EL, Orme AE, Vasquez S, Jarvik ME. 1978. Memory facilitating and anti-amnesic effects of corticosteroids. Pharmacol Biochem Behav 8: 81-87. doi:10.1016/0091-3057(78)90127-2

Gagnon SA, Wagner AD. 2016. Acute stress and episodic memory retrieval: neurobiological mechanisms and behavioral consequences. Ann N Y Acad Sci 1369: 55-75. doi:10.1111/nyas.12996

Gagnon SA, Waskom ML, Brown TI, Wagner AD. 2018. Stress impairs episodic retrieval by disrupting hippocampal and cortical mechanisms of remembering. Cereb Cortex doi:10.1093/cercor/bhy162.

Harris AP, Holmes MC, de Kloet ER, Chapman KE, Seckl JR. 2013. Mineralocorticoid and glucocorticoid receptor balance in control of HPA axis and behaviour. Psychoneuroendocrinology 38: 648-658. doi:10.1016/ j.psyneuen.2012.08.007

Hawley D, Leasure J. 2012. Region-specific response of the hippocampus to chronic unpredictable stress. Hippocampus 22: 1338-1349. doi:10.1002/ hipo. 20970

Henckens MJ, Hermans EJ, Pu Z, Joëls M, Fernández G. 2009. Stressed memories: how acute stress affects memory formation in humans. $J$ Neurosci 29: 10111-10119. doi:10.1523/JNEUROSCI.1184-09.2009

Het S, Ramlow G, Wolf OT. 2005. A meta-analytic review of the effect of acute cortisol administration on human memory.

Psychoneuroendocrinology 30: 771-884. doi:10.1016/j.psyneuen.2005.03 .005

Het S, Rohleder N, Schoofs D, Kirschbaum C, Wolf O. 2009. Neuroendocrine and psychometric evaluation of a placebo version of the 'Trier Social Stress Test'. Psychoneuroendocrinology 34: 1075-1086. doi:10.1016/j .psyneuen.2009.02.008

Holz E, Lass-Hennemann J, Streb M, Pfaltz M, Michael T. 2014. Effects of acute cortisol administration on perceptual priming of trauma-related material. PLoS One 9: e104864. doi:10.1371/journal.pone.0104864

Huffman DJ, Stark CE. 2017. The influence of low-level stimulus features on the representation of contexts, items, and their mnemonic associations. Neuroimage 155: 513-529. doi:10.1016/j.neuroimage.2017.04.019

Joëls M, Karst H, Alfarez D, Heine VM, Qin Y, van Riel E, Verkuyl M, Lucassen PJ, Krugers HJ. 2004. Effects of chronic stress on structure and cell function in rat hippocampus and hypothalamus. Stress 7: 221-231. doi:10.1080/10253890500070005

Joels M, Krugers H, Karst H. 2008. Stress-induced changes in hippocampal function. Prog Brain Res 167: 3-15. doi:10.1016/S0079-6123(07)67001-0

Kajantie E, Phillips DI. 2006. The effects of sex and hormonal status on the physiological response to acute psychosocial stress.

Psychoneuroendocrinology 31: 151-178. doi:10.1016/j.psyneuen.2005.07 .002

Kheirbek MA, Klemenhagen KC, Sahay A, Hen R. 2012. Neurogenesis and generalization: a new approach to stratify and treat anxiety disorders. Nat Neurosci 15: 1613-1620. doi:10.1038/nn.3262

Kirschbaum C, Pirke KM, Hellhammer DH. 1993. The 'Trier Social Stress Test'-a tool for investigating psychobiological stress responses in a laboratory setting. Neuropsychobiology 28: 76-81. doi:10.1159/ 000119004

Kirschbaum C, Kudielka BM, Gaab J, Schommer NC, Hellhammer DH. 1999. Impact of gender, menstrual cycle phase, and oral contraceptives on the activity of the hypothalamus-pituitary-adrenal axis. Psychosom Med 61: 154-162. doi:10.1097/00006842-199903000-00006

Kirwan CB, Stark CE. 2007. Overcoming interference: an fMRI investigation of pattern separation in the medial temporal lobe. Learn Mem 14: 625633. doi:10.1101/lm.663507

Kirwan CB, Jones CK, Miller MI, Stark CE. 2007. High-resolution fMRI investigation of the medial temporal lobe. Hum Brain Mapp 28: 959-966. doi:10.1002/hbm.20331

Kovacs GL, Telegdy G, Lissák K. 1976. 5-Hydroxytryptamine and the mediation of pituitary-adrenocortical hormones in the extinction of active avoidance behaviour. Psychoneuroendocrinology 1: 219-230. doi:10 .1016/0306-4530(76)90012-3

Kuhlmann S, Wolf OT. 2006. Arousal and cortisol interact in modulating memory consolidation in healthy young men. Behav Neurosci 120: 217. doi:10.1037/0735-7044.120.1.217

Lacy JW, Yassa MA, Stark SM, Muftuler LT, Stark CE. 2011. Distinct pattern separation related transfer functions in human CA3/dentate and CA1 revealed using high-resolution fMRI and variable mnemonic similarity. Learn Mem 18: 15-18. doi:10.1101/lm.1971111

Leutgeb S, Leutgeb JK, Treves A, Moser MB, Moser EI. 2004. Distinct ensemble codes in hippocampal areas CA3 and CA1. Science 305: 12951298. doi:10.1126/science. 1100265

Leutgeb JK, Leutgeb S, Moser MB, Moser EI. 2007. Pattern separation in the dentate gyrus and CA3 of the hippocampus. Science 315: 961-966. doi:10.1126/science.1135801
McCullough AM, Yonelinas AP. 2013. Cold-pressor stress after learning enhances familiarity-based recognition memory in men. Neurobiol Learn Mem 106: 11-17. doi:10.1016/j.nlm.2013.06.011

McCullough AM, Ritchey M, Ranganath C, Yonelinas A. 2015. Differential effects of stress-induced cortisol response on recollection and familiarity-based recognition memory. Neurobiol Learn Mem 123: 1-10. doi:10.1016/j.nlm.2015.04.007

McEwen BS. 1999. Stress and hippocampal plasticity. Аnпu Rev Neurosci 22: 105-122. doi:10.1146/annurev.neuro.22.1.105

McGaugh JL, Roozendaal B. 2002. Role of adrenal stress hormones in forming lasting memories in the brain. Curr Opin Neurobiol 12: 205-210. doi:10.1016/S0959-4388(02)00306-9

Petrowski K, Herold U, Joraschky P, Wittchen HU, Kirschbaum C. 2010. A striking pattern of cortisol non-responsiveness to psychosocial stress in patients with panic disorder with concurrent normal cortisol awakening responses. Psychoneuroendocrinology 35: 414-421. doi:10.1016/j .psyneuen.2009.08.003

Rimmele U, Domes G, Mathiak K, Hautzinger M. 2003. Cortisol has different effects on human memory for emotional and neutral stimuli. Neuroreport 14: $2485-2488$. doi:10.1097/00001756-200312190-00038

Rimmele U, Besedovsky L, Lange T, Born J. 2015. Emotional memory can be persistently weakened by suppressing cortisol during retrieval. Neurobiol Learn Mem 199: 102-107. doi:10.1016/j.nlm.2015.01.010

Rohleder N, Schommer NC, Hellhammer DH, Engel R, Kirschbaum C. 2001. Sex differences in glucocorticoid sensitivity of proinflammatory cytokine production after psychosocial stress. Psychosom Med 63: 966972. doi:10.1097/00006842-200111000-00016

Roozendaal B. 2002. Stress and memory: opposing effects of glucocorticoids on memory consolidation and memory retrieval. Neurobiol Learn Mem 78: 578-595. doi:10.1006/nlme.2002.4080

Sandi C. 2003. Glucocorticoid involvement in memory consolidation. Rev Neurol 37: 843-848.

Sandi C, Pinelo-Nava MT. 2007. Stress and memory: behavioral effects and neurobiological mechanisms. Neural Plast 2007: 78970. doi:10.1155/ $2007 / 78970$

Shields G, Sazma M, McCullough A, Yonelinas A. 2017. The effects of acute stress on episodic memory: a meta-analysis and integrative review. Psychol Bull 143: 636-675. doi:10.1037/bul0000100

Smeets T, Otgaar H, Candel I, Wolf OT. 2008. True or false? Memory is differentially affected by stress-induced cortisol elevations and sympathetic activity at consolidation and retrieval. Psychoneuroendocrinology 33: 1378-1386. doi:10.1016/j.psyneuen.2008 .07 .009

Smyth J, Ockenfels MC, Porter L, Kirschbaum C, Hellhammer DH, Stone AA. 1998. Stressors and mood measured on a momentary basis are associated with salivary cortisol secretion. Psychoneuroendocrinology 23: 353-370. doi:10.1016/S0306-4530(98)00008-0

Spielberger CD, Sydeman SJ. 1994. State-trait anxiety inventory and state-trait anger expression inventory. In The use of psychological testing for treatment planning and outcome assessment (ed. Maruish ME), pp. 292321. Lawrence Erlbaum Associates, Inc, Hillsdale, NJ.

Stark SM, Stark CE. 2017. Age-related deficits in the mnemonic similarity task for objects and scenes. Behav Brain Res 333: 109-117. doi:10.1016/j .bbr.2017.06.049

Stark SM, Yassa MA, Lacy JW, Stark CE. 2013. A task to assess behavioral pattern separation (BPS) in humans: data from healthy aging and mild cognitive impairment. Neuropsychologia 51: 2442-2449. doi:10.1016/j .neuropsychologia.2012.12.014

Stark SM, Stevenson R, Wu C, Rutledge S, Stark CE. 2015. Stability of age-related deficits in the mnemonic similarity task across task variations. Behav Neurosci 129: 257. doi:10.1037/bne0000055

Tran TT, Speck CL, Pisupati A, Gallagher M, \& Bakker A. 2017. Increased hippocampal activation in ApoE-4 carriers and non-carriers with amnestic mild cognitive impairment. Neuroimage 13: 237-245. doi:10 $.1016 /$ j.nicl.2016.12.002

Wilson IA, Ikonen S, Gallagher M, Eichenbaum H, Tanila H. 2005. Age-associated alterations of hippocampal place cells are subregion specific. J Neurosci 25: 6877-6886. doi:10.1523/JNEUROSCI.1744-05 .2005

Wolf OT. 2003. HPA axis and memory. Best Pract Res Clin Endocrinol Metab 17: 287-299. doi:10.1016/S1521-690X(02)00101-X

Yassa MA, Stark SM, Bakker A, Albert MS, Gallagher M, Stark CE. 2010. High-resolution structural and functional MRI of hippocampal CA3 and dentate gyrus in patients with amnestic mild cognitive impairment NeuroImage 51: 1242-1252. doi:10.1016/j.neuroimage.2010.03.040

Received October 1, 2018; accepted in revised form March 8, 2019. 


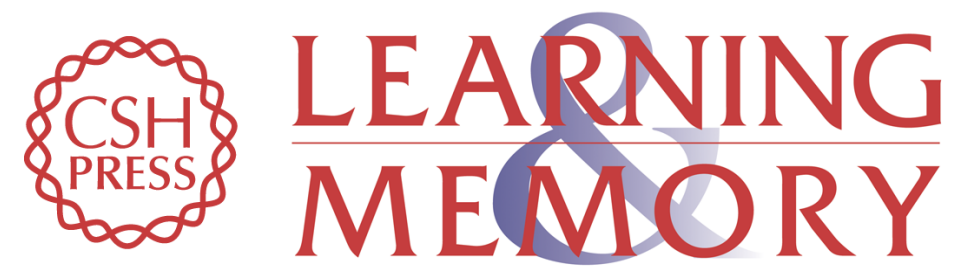

\section{Acute stress-induced cortisol elevation during memory consolidation enhances pattern separation}

Alice Jiang, Tammy T. Tran, Farrah N. Madison, et al.

Learn. Mem. 2019, 26:

Access the most recent version at doi:10.1101/Im.048546.118

References This article cites 57 articles, 9 of which can be accessed free at: http://learnmem.cshlp.org/content/26/4/121.full.html\#ref-list-1

Creative This article is distributed exclusively by Cold Spring Harbor Laboratory Press for the Commons License first 12 months after the full-issue publication date (see

http://learnmem.cshlp.org/site/misc/terms.xhtml). After 12 months, it is available under a Creative Commons License (Attribution-NonCommercial 4.0 International), as described at http://creativecommons.org/licenses/by-nc/4.0/.

Email Alerting Receive free email alerts when new articles cite this article - sign up in the box at the Service top right corner of the article or click here. 